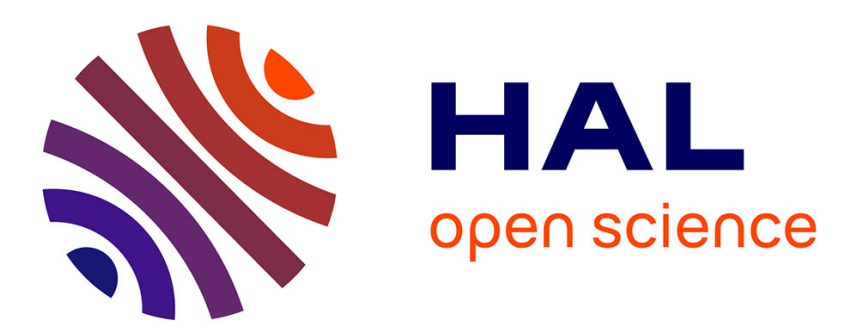

\title{
Application of Bicoherence Analysis in Study of Wave Interactions in Space Plasma
}

Dominique Lagoutte, François Lefeuvre, J. Hanasz

\section{To cite this version:}

Dominique Lagoutte, François Lefeuvre, J. Hanasz. Application of Bicoherence Analysis in Study of Wave Interactions in Space Plasma. Journal of Geophysical Research Space Physics, 1989, 94 (A1), pp.435-442. 10.1029/JA094iA01p00435 . insu-02880211

\section{HAL Id: insu-02880211 https://hal-insu.archives-ouvertes.fr/insu-02880211}

Submitted on 24 Jun 2020

HAL is a multi-disciplinary open access archive for the deposit and dissemination of scientific research documents, whether they are published or not. The documents may come from teaching and research institutions in France or abroad, or from public or private research centers.
L'archive ouverte pluridisciplinaire HAL, est destinée au dépôt et à la diffusion de documents scientifiques de niveau recherche, publiés ou non, émanant des établissements d'enseignement et de recherche français ou étrangers, des laboratoires publics ou privés. 


\title{
Application of Bicoherence Analysis in Study of Wave Interactions in Space Plasma
}

\author{
D. LAGOUTTE and F. LEFEUVRE \\ Laboratoire de Physique et Chimie de l'Environnement, CNRS, Orléans, France
}

\section{J. HANASZ}

Copernicus Astronomical Center, Torun, Poland

\begin{abstract}
A spectral analysis at the second order (power spectrum) loses the phase information among the different Fourier components. To retain this information, the bispectrum (third order) and/or the bicoherence (normalized bispectrum) are calculated. Application to simulated data, shows the dependence of the bispectrum to amplitudes of involved waves and of the bicoherence to signal-to-noise ratio. Bicoherence technique is applied in the analysis of harmonics produced by an electronic receiver, as well as in the investigation of phase coherence between a ground-transmitter signal, a natural ELF emission near the proton gyrof requency, and the sidebands around the carrier. Strong arguments are provided that the sidebands are generated by a parametric interaction between the transmitter signal and the ELF emission.
\end{abstract}

\section{INTRODUCTION}

Several suggestions of possible wave-wave interactions in the ionosphere and the magnetosphere have been reported. For example, Riggin and Kelley [1982] have suggested that parametric decay of whistler mode waves into lower hybrid waves may occur for strong natural or artificial emissions. Koons et al. [1987] have observed the generation of ion acoustic waves from the decay of Langmuir waves near the electron plasma frequency. Wave-wave interactions have been proposed to explain the auroral kilometric radiation, specifically the narrow bands [e.g., Roux and Pellat, 1979; Grabbe et al., 1980; Altman et al., 1987], although on the other hand the maser process has been admitted for several years as being responsible for this radiation [Wu and Lee, 1979]. A bicoherence analysis of the waveform observed with ARCAD 3, supports the suggestion that a nonlinear-mode coupling between the transmitter pulse and ELF emission can produce the sidebands around the transmission frequency [Tanaka et al., 1987].

The use of bicoherence technique has also appeared to be very successful in studies of wave phenomena in various media, such as harmonic generation of ocean-surface gravity waves [Elgar and Guza, 1985], brain wave emissions [Huber et al., 1971], nonlinear transfer of energy in turbulent flows [Lii et al., 1976; Van Atta, 1979], and nonlinear interaction between modes of density fluctuations in laboratory plasmas [Kim and Powers, 1979; Kim et al., 1980]. Furthermore, the considerable amount of literature regarding higher-order spectral analysis is so well known that it barely needs mention here.

The higher-order spectral analysis appears to be unique in attempts to answer the question of the presence of multiwave interaction processes, because phase information is not lost in it. This is why, in the era of fast analog-to-digital converters and large random-access memories, the method of bicoherence analysis is expected to be increasingly used in studies of wave generation in space plasmas.

Numerous difficulties exist with the application of the bicoherence technique to space experiments. First, full waveforms, sometimes in very large bands, must be teleme-

Copyright 198.9 by the American Geophysical Union.

Paper number 7A9462.

0148-0227/89/007A-9462\$05.00 tered to the ground, a condition that is generally incompatible with the telemetry capacities. Moreover, satellite interferences and nonlinearities produced by the on-board electronics, have a tendency to mask the natural phenomena. Second, the resonance condition for a three-wave nonlinear interaction to take place $\left(f_{3}=f_{1}+f_{2}, \mathbf{K}_{3}=\mathbf{K}_{1}+\mathbf{K}_{2}\right)$ is expected to be satisfied in the source region only. Third, the bicoherence analysis only allows the detection of quadratic nonlinear interactions, whereas a higher order of interactions may be produced in space plasmas. The present study shows that, despite all those difficulties, the bicoherence technique does detect three- wave nonlinear interactions in space.

The aims of this paper are to describe the bicoherence analysis as applied to the wave data collected in space experiments, to illustrate how the method can be applied to the specific data obtained in the wave experiment of ARCAD 3, and to show the advantages and limits of the method.

The plan of the paper is as follows. First, the basic definitions of the bispectrum and bicoherence are recalled (section 2). Then, simulated data are analyzed to show how estimators of the bispectrum and bicoherence can describe the phase coherence of the interacting signals, and to highlight the problems that arise when one or the other is used (section 3). Section 4 is devoted to the analysis of experimental data recorded on ARCAD 3, characteristic for the sidebands accompanying the VLF transmission in the presence of ELF emission. Conclusions are presented in section 5.

\section{DEFINITIONS AND PROPERTIES}

\subsection{Basic Definitions}

Let $x(t)$ be a real and stationary signal with zero mean value and $X(f)$ its Fourier transform. Hereafter, $X_{k}$, the Fourier component at the frequency $f_{k}$ is noted as

$$
\mathrm{X}_{\mathrm{k}}=\int_{0}^{T} x(t) \exp \left(-2 \pi j f_{k} t\right) d t
$$

The definitions of the second moment of the time series concern autocorrelation and the power spectrum. The autocorrelation for the record $(i)$ at the time lag $r$ is defined by

$$
R^{(i)}(\tau)=\frac{1}{T} \int_{0}^{T} x^{(i)}(t) x^{(i)}(t+\tau) d t
$$


The power spectrum at the frequency $f_{k}$ is the Fourier transform of the autocorrelation

$S(k)=S\left(f_{k}\right)=\int_{-T}^{T} R(\tau) \exp \left(-2 \pi j f_{k} \tau\right) d \tau$

where $R(\tau)=E\left\{R^{(i)}(\tau)\right\}$.

An alternate equivalent way is [Bendat and Piersol, 1971]

$$
S(k)=\lim _{T \rightarrow \infty} \frac{1}{T} E\left(X_{k}^{(i)} X_{k}^{(i)^{*}}\right)
$$

where the average $E\{\}$ is taken over $i=1, \ldots M$ successive data records, and $X_{k}^{*}$ is the complex conjugate of $X_{k}$.

Similarly, the third moment at the time lags $\tau_{1}$ and $\tau_{2}$ is

$$
R\left(\tau_{1}, \tau_{2}\right)=E\left\{\frac{1}{T} \int_{0}^{T} x^{(i)}(t) x^{(i)}\left(t+\tau_{1}\right) \cdot x^{(i)}\left(t+\tau_{2}\right) d t\right\}
$$

and, by definition, the bispectrum at the frequencies $f_{k}$ and $f_{l}$ is the two-dimensional Fourier transform of $R\left(r_{1}, r_{2}\right)$ :

$$
\begin{aligned}
B(k, l)= & B\left(f_{k^{\prime}}, f_{l}\right)=\int_{-T}^{T} \int_{-T}^{T} R\left(\tau_{1} \tau_{2}\right) \\
& . \exp \left(-2 \pi j\left(f_{k} \tau_{1}+f_{l} \tau_{2}\right)\right) d \tau_{1} d \tau_{2}
\end{aligned}
$$

Now, the bispectrum can be defined as a product of three Fourier components [Kim and Powers, 1979]:

$$
B(k, l)=\lim _{T \rightarrow \infty} \frac{1}{T} E\left\{X_{k}^{(i)} X_{l}^{(i)} X_{k+l}^{(i)}\right\}
$$

But this definition is only valid if the sum of the frequencies of the three Fourier components is zero, which means

$$
f_{k \pm l}=f_{k} \pm f_{l}
$$

where $f_{k \pm}$ is the frequency of the product wave in case of coupling. The bicoherence spectrum is a normalized bispectrum:

$$
\mathrm{b}^{2}(k, l)=\frac{\left|E\left\{x_{k} x_{l} x_{k+l}^{*}\right\}\right|^{2}}{E\left\{\left|X_{k} x_{l}\right|^{2}\right\} E\left\{\left|X_{k+l}\right|^{2}\right\}}
$$

where $|B|$ is the modulus of $B$; and $0 \leq b(k, l) \leq 1$.

The variance of $b(k, l)$ is expressed as

$$
\operatorname{var}[b(k, l)] \simeq \frac{1}{M}\left[1-b^{2}(k, l)\right]
$$

For more details about the above definitions, see Kim and Powers [1979].

The bispectrum and bicoherence are important tools to investigate the phase coherence between three Fourier components of frequencies $f_{k}, f_{l}, f_{k+l}$. If the third wave at the frequency $f_{k+l}=f_{k}+f_{l}$ is spontaneously emitted, the phase of the Fourier component $X_{k+l}$ will be independent of the phase of the two others, $X_{k}$ and $X_{l}$, and the values of bispectrum (7) and bicoherence (10) will be zero. On the other hand, if the wave at the frequency $f_{k+l}$ is phase coherent with the two other waves, the values of bispectrum and bicoherence will be nonzero.

\subsection{Computation Region}

The frequencies $f_{k}, f_{l}$ and $f_{k+l}$ are limited by the maximum frequency $f_{N}$ of the studied signal. The total region of computation of bispectrum and bicoherence is the hexagonal region shown in Figure 1a. But, due to the symmetry relations

$$
B(k, l)=B(l, k)=B^{*}(-k,-l)=B^{*}(-k, k+l)
$$

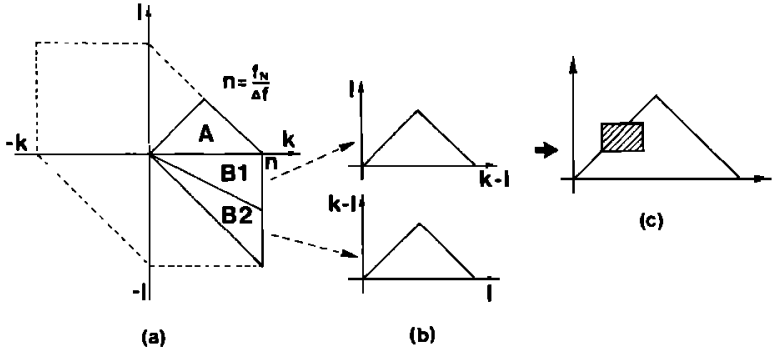

Fig. 1. Region of computation of bispectrum and bicoherence spectrum. (a) Hexagonal area (dashed line) is the definition region. Due to symmetry relations, the bispectrum is calculated over the triangle $A$, and the bicoherence over the triangle $\mathrm{A}$ for sum interaction $(k, l \rightarrow k+l)$, and over the triangles B1, B2 for difference interaction $(k,-l \rightarrow k-$ $l$ ). (b) Coordinate transformation for the region of computation of bicoherence spectrum for difference interaction. (c) Restricted area of computation (hatched rectangle) in case of memory limitation.

one can limit the computation of the bispectrum on the $A$ region, which is the triangle defined by

$$
0 \leq f_{l} \leq f_{N} / 2 \quad f_{l} \leq f_{k} \leq f_{N}-f_{l}
$$

This is somewhat different for the bicoherence. Due to the normalization in the bicoherence definition (9), a symmetry has been lost and we only have

$$
b(k, l)=b(l, k)=b(-k,-l)
$$

Then, the restricted region for the bicoherence computation is formed by the A, B1 and B2 areas in Figure 1a. The A region is for the sum interaction $k, l \rightarrow k+l$, and the $(\mathrm{B} 1+\mathrm{B} 2)$ region is for the difference interaction $k,-l \rightarrow k-l$. (B1 : $k$, $-l \rightarrow k-l$ with $k-l \geq l$, and $\mathrm{B} 2: k,-l \rightarrow k-l$ with $k-l \leq l)$ with an appropriate change of variables, whereas the Bl and $B 2$ regions can be represented like the $A$ region (Figure lb). The shape of the three areas is now the same, but with different variables on the axes.

When high-f requency resolution is needed, the problem of computer memory arises, because the area of the triangle is $N / 2 . N / 4=N^{2} / 8$, where $N$ is the number of samples in a record. The way to overcome this problem, is to select a restricted area of computations as shown by the hatched rectangle in Figure lc.

\section{APPLICATION TO SIMULATED DATA}

A test signal is considered in such a manner that

$$
\begin{aligned}
& x(t)=2 \cos \left(2 \pi f_{1} t+\psi_{1}\right)+\cos \left(2 \pi f_{2} t+\psi_{2}\right) \\
& +\cos \left(2 \pi f_{3} t+\psi_{3}\right)+\cos \left(2 \pi f_{4} t+\psi_{4}\right)+N(t)
\end{aligned}
$$

with $f_{1}=50 \mathrm{~Hz}, f_{2}=100 \mathrm{~Hz}, f_{3}=150 \mathrm{~Hz}, f_{4}=250 \mathrm{~Hz}$ and a sampling frequency of $2000 \mathrm{~Hz}$. The phases $\psi_{1}, \psi_{2}, \psi_{3}$ are randomly distributed over $[-\pi,+\pi]$ and the phase $\psi_{4}=\psi_{2}+\psi_{3} . N(t)$ is a white gaussian noise.

With the phase coherence for the waves at frequencies $f_{2}$ $f_{3}, f_{4}$, we expect a nonzero value for the bispectrum and bicoherence at these frequencies $\left(B\left(f_{3}, f_{2}\right)\right.$ and $\left.b\left(f_{3}, f_{2}\right)\right)$ and a zero value otherwise.

The autospectrum of the signal $x(t)$ is shown in Figure 2a, and the bispectrum and bicoherence spectrum in Figures $2 b$ and $2 c$. The bispectrum has two peaks, whereas only one was expected. The peak at frequencies $f_{2}$ and $f_{3}$ is correct, because a phase coherence of the waves exists at frequencies 


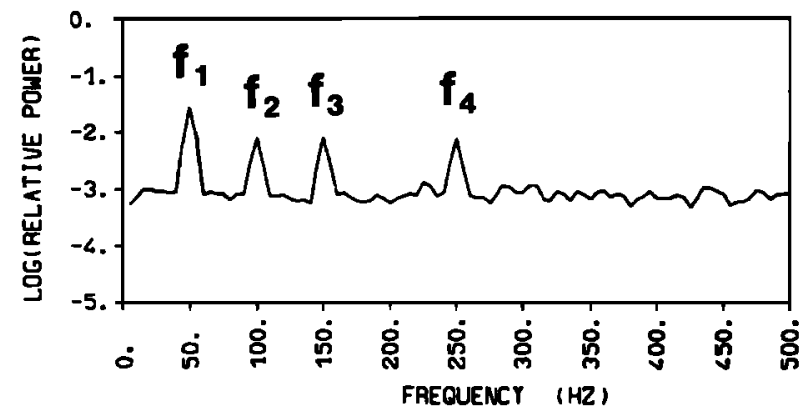

(a)

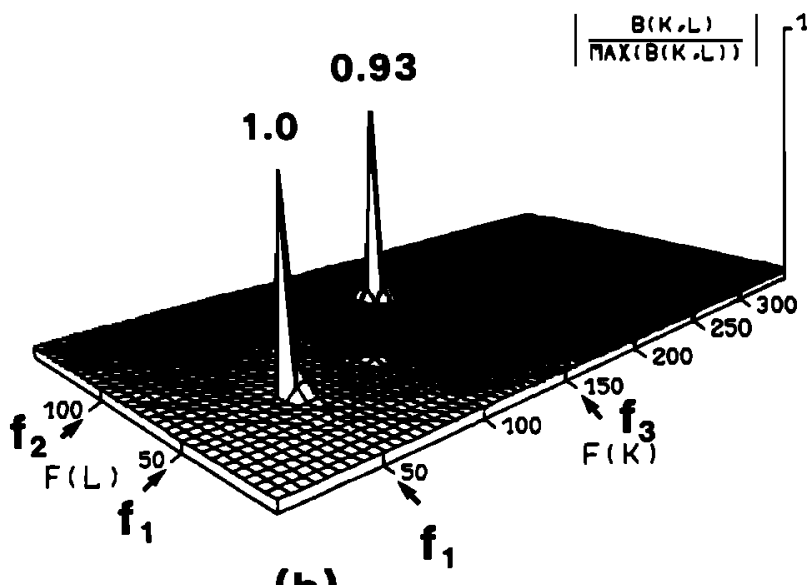

(b)

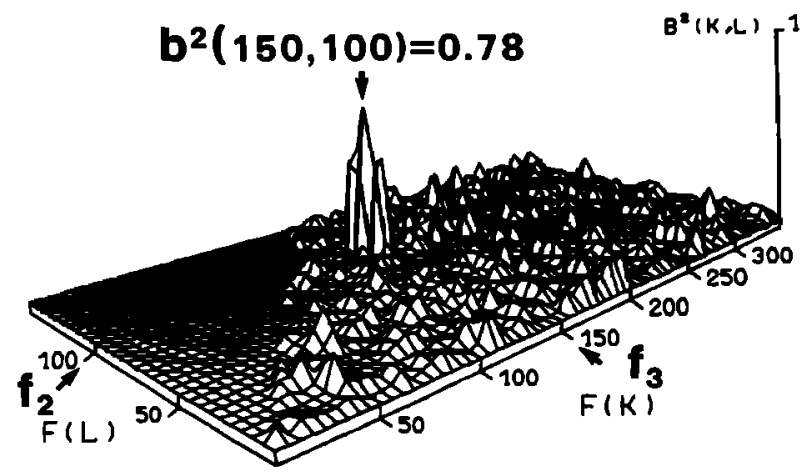

(c)

Fig. 2. Analysis of test signal defined by equation (13). The FFT is performed with $N=400$ samples in each record, and the average is taken over $M=30$ data records. Frequency resolution is $5 \mathrm{~Hz}$. (a) Autospectrum. (b) Bispectrum. (c) Bicoherence spectrum.

$f_{2}, f_{3}$ and their sum $f_{4}=f_{2}+f_{3}$. However, the peak $B\left(f_{1}, f_{1}\right)$ has no physical meaning. Though $f_{2}=f_{1}+f_{1}$, there is no phase coherence supposed for these signals. The reason for this second peak is that the bispectrum is very sensitive to the amplitudes of the involved spectral components. This example shows clearly that, even for incoherent signals, the value of the bispectrum can be significant, if the averaging is performed on too small a number of records (in this case $M=$ 30). The conclusion must be that the use of the bispectral estimator can be misleading; for this reason, the following analysis will deal only with the bicoherence analysis.

In Figure $2 c$, only the expected peak $b^{2}\left(f_{3}, f_{2}\right)$ is present. Although the waves at frequencies $f_{2}, f_{3}, f_{4}=f_{2}+f_{3}$ are phase coherent, the maximum value of the bicoherence estimator is only $b^{2}\left(f_{3}, f_{2}\right)=0.78$ instead of 1 . This is due to the noise $N(t)$, added to the test signal. Although, theoretically, the bispectrum is independent of noise [Lagoutte, 1983 ], the bicoherence is very sensitive to $S / N$ due to the normalization. If this ratio is low, the value of bicoherence estimated with a relatively small number of data records ( $M$ < 50) can be non significant, even in the case of phase coherence. To recover significant value the number of data records must be increased (up to several hundred) at least if the signal is stationary over a sufficiently long time interval.

Another problem in experimental applications of bicoherence technique is how to digitize the signal, eight-bit samples being commonly used. Our simulations show that, even with three-bit sampling, the bicoherence spectrum is practically identical to the one obtained with the eight-bit sampling, whereas with two-bit sampling spurious peaks appear in the bicoherence spectrum.

In conclusion to this section, it can be said that the use of bispectrum analysis can be subject to wrong interpretation. Bicoherence can only be used if the waves are not embedded in noise. Digitization of a signal to three-bit or larger samples will not distort the bicoherence spectra.

\section{APPLICATION TO ARCAD 3 DATA}

The data were recorded on board the AUREOL 3 satellite, which formed part of the Soviet-French project ARCAD 3 for the study of the ionosphere and magnetosphere. In this case, the working mode C2 of the VLF experiment [Berthelier et al., 1982] was of interest. In this mode, one electric or one magnetic component in a $70-\mathrm{Hz}$ to $16-\mathrm{kHz}$ bandwidth was telemetered during a 4-s time interval. On a spectrogram (not shown in this paper) of the $E_{H}$ component ( $E_{H}$ is in a plane $11^{\circ}$ of $f$ the plane perpendicular to $\mathrm{B}_{\mathrm{o}}$ ) recorded on August 11,1982, an ELF noise around $500 \mathrm{~Hz}$ was observed, as well as three pulses emitted by the transmitter of the Alpha station in the USSR. (geographic coordinates $50.5^{\circ} \mathrm{N}, 137^{\circ} \mathrm{E}$ ). The three different frequencies are $11.9 \mathrm{kHz}, 12.65 \mathrm{kHz}$, and $14.9 \mathrm{kHz}$, and each pulse has a duration of $400 \mathrm{~ms}$. A spectral broadening of the carrier frequency was also observed. According to Tanaka et al. [1987], this phenomenon can be described in two types: (1) without sideband structure; (2) with sideband structure, observed only on the $E_{H}$ component. The power spectra presented in Figures $4 \mathrm{a}$ and $5 \mathrm{a}$, clearly show the sideband structure around the carrier frequency (respectively 11.9 $\mathrm{kHz}$ and $12.65 \mathrm{kHz}$ ) and also a strong ELF emission at and above $500 \mathrm{~Hz}$. In section 4.2 , the phase coherence among these different waves will be investigated by means of the bicoherence estimator.

When analyzing ARCAD 3 data, interference is commonly encountered, which is generated by the spacecraft system and shows harmonic structures. Here, bicoherence analysis will be used to answer the question whether these structures are phase coherent.

\subsection{Analysis of Harmonics}

Figure $3 a$ represents an autospectrum of the $E_{H}$ component from 0 up to $15 \mathrm{kHz}$. The VLF-transmitter signal at 11.9 $\mathrm{kHz}$ and a number of harmonics of $\simeq 800 \mathrm{~Hz}$ can be seen, and Figure $3 b$ shows the map of their bicoherence spectrum. One can conclude from it the following :

1. Harmonics are phase coherent with a maximum value of the bicoherence $b^{2}(2.4,1.6)=0.92$, which indicates that some nonlinear process must be operating at the fundamental frequency of $\simeq 800 \mathrm{~Hz}$.

2. The horizontal, vertical and $45^{\circ}$ structures of weak peaks in the bicoherence spectrum can be easily explained if 


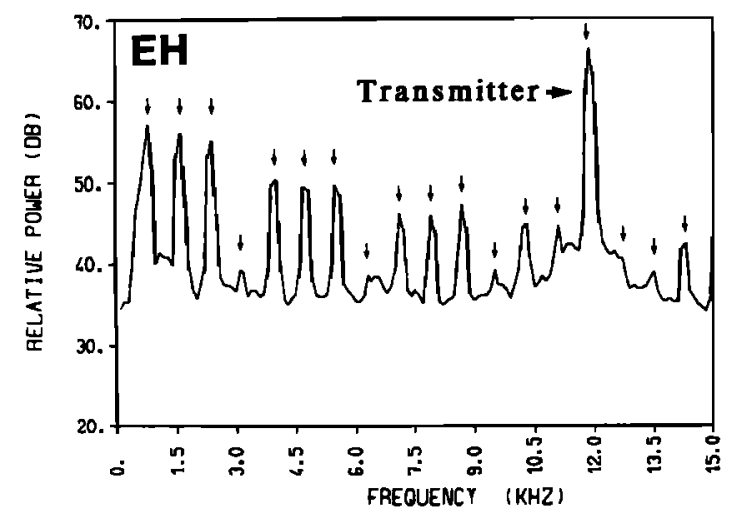

(a)

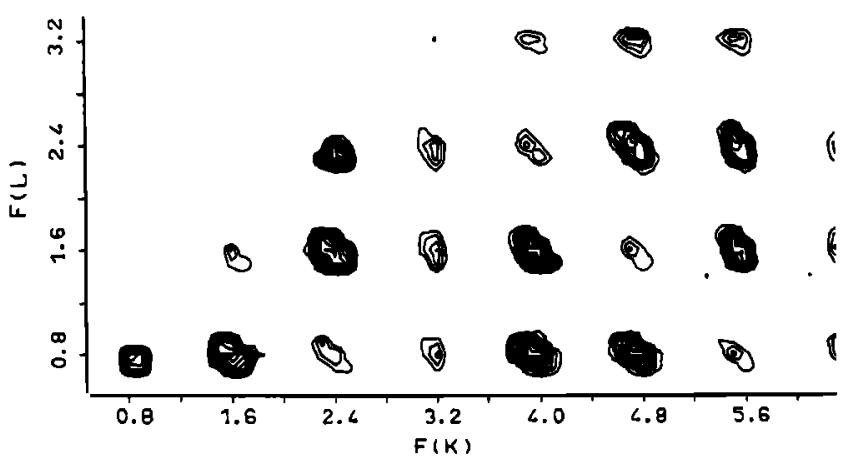

(b)

Fig. 3. Spectral and bicoherence analyses of the electric $E_{H}$ component recorded on September 29, 1982, at 2225:6.5 UT. Analyses are performed with $N=500$ samples in each record and the average is taken over $M=38$ data records. Frequency resolution is $100 \mathrm{~Hz}$. (a) Autospectrum. Arrows indicate harmonics of $\simeq 800 \mathrm{~Hz}$. (b) Bicoherence spectrum.

one takes into account that fourth, eigth, twelth, etc., peaks in the power spectrum are of low magnitude. As the bicoherence estimator is very sensitive to $\mathrm{S} / \mathrm{N}$, its corresponding values along lines where $f_{k}, f_{l}, f_{k+l}$ are multiples of the fourth harmonic, will be weaker than the ones at points corresponding to other harmonics.

\subsection{Analysis of Spectral Broadening of the Transmitter Signal}

Five cases of spectral broadening, characteristic for sidebands around the carrier at $E_{H}$ component have been already analyzed [Tanaka et al., 1987]. For the purpose of this paper, only two cases were selected, which appeared to be the best for illustrating how the bicoherence analysis works.

4.2.1. Transmitter frequency $11.9 \mathrm{kHz}$, time 0423:11.4. The power spectrum presented in Figure 4a shows the two distinct sidebands, the lower being at $11.4 \mathrm{kHz}$ and the upper at $12.4 \mathrm{kHz}$. Within the low-f requency range, a peak of ELF emission is observed at $500 \mathrm{~Hz}$. Since these types of waves satisfy the frequency condition for parametric interaction, a coupling between transmitter and ELF waves is expected, which produces the sideband emissions. Therefore the region of interest is limited to the rectangle of frequency intervals : 9.5 to $15 \mathrm{kHz}$ and 0 to $3 \mathrm{kHz}$. As explained in section 2, the studies of sum interaction and difference interaction are done on two different plots. The bicoherence spectrum for the sum interaction (upper sideband) is displayed in Figures $4 b$ and $4 c$, and for the difference (lower sideband) in Figures $4 \mathrm{~d}$ and $4 \mathrm{e}$. The main peak, $b^{2}$ $(11.9,0.5)=0.65$, shows a phase coherence between the transmitter $(11.9 \mathrm{kHz})$, the ELF emission $(0.5 \mathrm{kHz})$ and the upper sideband $(12.4 \mathrm{kHz})$. The value of the bicoherence is significant, though $\mathrm{S} / \mathrm{N}$ in the power spectrum is low at sideband frequencies. The bicoherence estimator for the lower sideband is $b^{2}(11.9,-0.5)=0.53$. Again, even though the bicoherence value is lower than previously, it can be concluded that the transmitter signal, ELF emission and lower sideband are phase coherent. The interpretation of peaks of minor bicoherence value (secondary peaks) will be given in the following subsection.

4.2.2. Transmitter frequency $12.65 \mathrm{kHz}$, time 0423:28.8. The power spectrum is presented in Figure $5 \mathrm{a}$, bicoherence results for sum interactions (upper sideband) in Figures $5 b$ and $5 \mathrm{c}$, and for difference interaction (lower sideband) in Figures $5 \mathrm{~d}$ and $5 \mathrm{e}$. The maximum values are $b^{2}(12.7,0.5)=$ 0.65 and $b^{2}(12.6,-0.5)=0.68$, which means that here again phase coherence exists for all three types of waves.

In all the bicoherence maps secondary peaks can be identified. For instance, in Figure $5 \mathrm{c}$, for sum interaction, three secondary peaks exist at $f_{k}=11.7,12.2$, and $13.2 \mathrm{kHz}$ and the main peak at $12.7 \mathrm{kHz}$, the latter having been discussed in the preceding subsection. The peak $b^{2}(12.2,0.5)$ indicates a phase coherence for the lower sideband, ELF emission and transmitter. Physically, the product wave of interaction is the lower sideband and thus the correct denominator in the normalization (formula (9)) is $E\left\{\mid X_{12.6}\right.$ $\left.X_{0.5} \mid{ }^{2}\right)$. E $\left(\left|X_{12.1}\right|{ }^{2}\right\}$, which has been used for the corresponding peak in Figure 5e. To obtain the result in Figure 5c, looking for sum interąction, one normalizes with the denominator $E\left\{\left|X_{12.2} X_{0.5}\right|^{2}\right\} \cdot E\left\{\left|X_{12.7}\right|^{2}\right\}$, which is incorrect because the carrier is not a product wave.

Now the two other secondary peaks of bicoherence spectrum at $f_{k}=11.7$ and $13.2 \mathrm{kHz}$ (Figure 5c) should be considered. At these frequencies, faint sidebands can be identified in the power spectrum (Figure 5a), which will be called the secondary sidebands. These bands are phase coherent with the primary sidebands and ELF emission, which can be concluded from the bicoherence spectrum $\left(f_{k+2 l}=f_{k+l}+f_{l}, f_{k-2 l}=f_{k-l}-f_{l}\right)$. Though one can expect phase coherence between the carrier and the secondary sidebands, the bicoherence analysis is not able to prove it directly, because the frequency condition (equation (8)) is not satisfied.

4.2.3. Discussion. Two of the five cases of the sideband emissions, which appear in the power spectrum in the presence of transmitter emission and ELF noise, have been presented in this paper to illustrate the application of the bicoherence technique to wave experiments in space. According to Tanaka et al. [1987], the sidebands can be interpreted in terms of the three-wave interactions of plasma waves, whereas the electronic receiver is suggested to be excluded as a source of a nonlinear process between the transmitter and ELF signals.

The use of the momentum conservation law $\left(\mathbf{K}_{k} \pm \mathbf{K}_{l}=\right.$ $\mathbf{K}_{k+l}$ ) could confirm Tanaka's et al.'s interpretation. However, this is impossible with these data, since only one electric or one magnetic component of the wave field can be measured at a given time. It can, however, be expected that in this type of parametric process the energy of the secondary waves observed at sideband frequencies is produced at the expense of the primary waves of the carrier and ELF emission. But, due to the lack of knowledge about the transfer function of the receiving equipment, it was not possible to verify quantitatively the power-density balance for the suggested process. Nevertheless, a strong confirmation was obtained that the sidebands have a natural origin and are produced in the parametric process. In Figures 6a and $6 \mathrm{~b}$, time dependencies are presented for the powers received at the frequencies of interacting waves with the transmitter frequency at 11.9 and $12.65 \mathrm{kHz}$, respectively. 


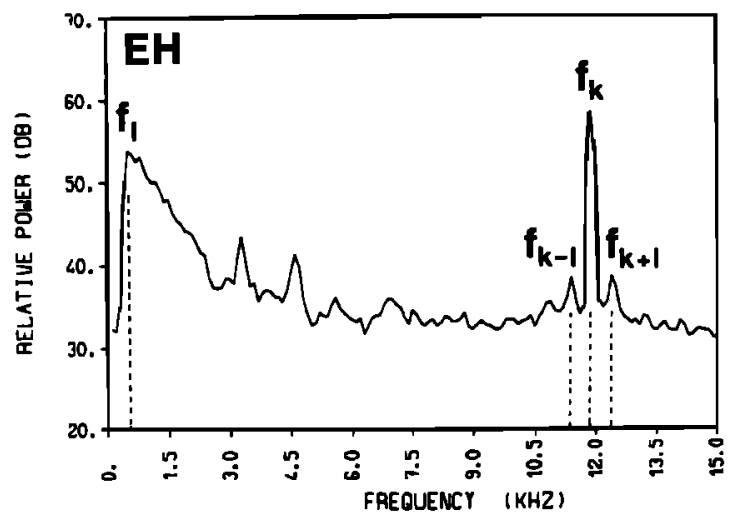

(a)

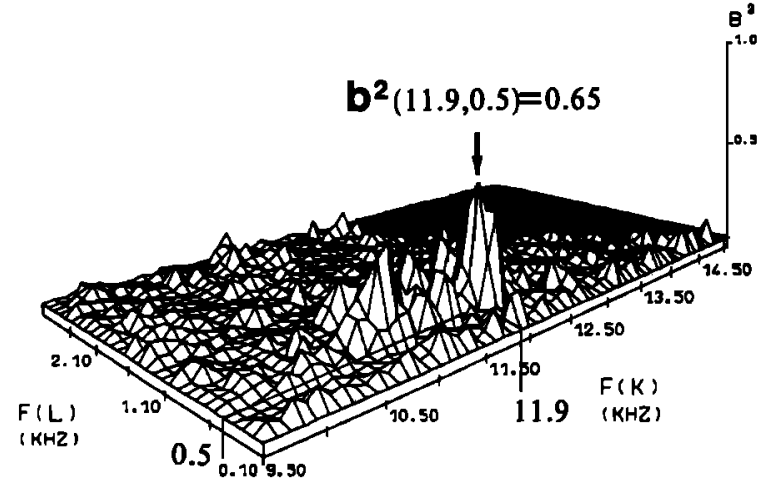

(b)

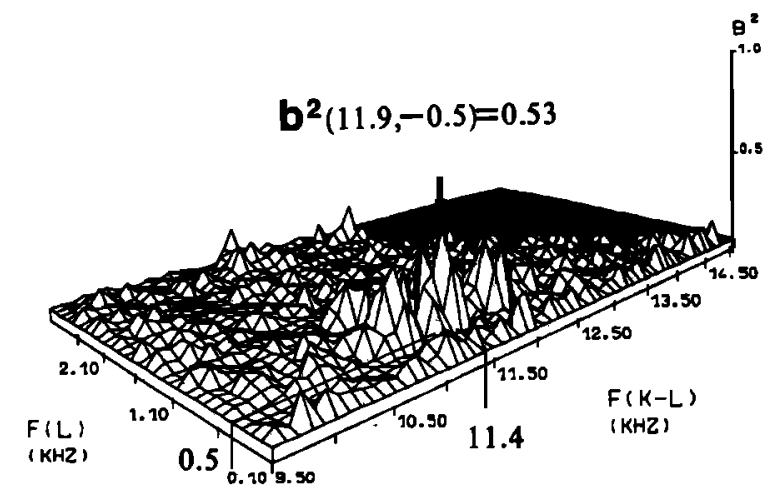

(d)

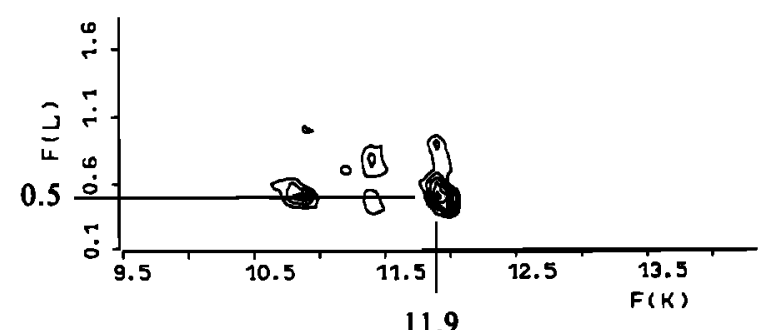

(c)

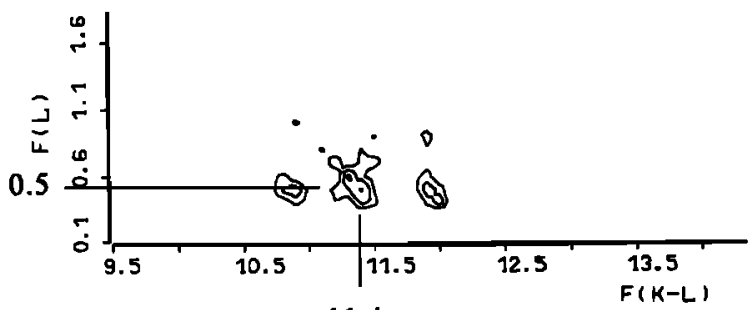

11.4 (e)

Fig. 4. Spectral and bicoherence analyses of the $E_{H}$ component recorded on August 11, 1982, at 0423:11.4 UT. The satellite coordinates are altitude $1178 \mathrm{~km}$, geographic latitude $46^{\circ} 7$, geographic longitude $135^{\circ} 5$. Analyses are performed with $N=500$ samples in each record and the average is taken over $M=38$ data records. Frequency resolution is $100 \mathrm{~Hz}$. (a) Autospectrum. The frequencies $f_{k^{\prime}} f_{l} f_{k+l^{\prime}} f_{k-l}$ represent, respectively, the frequencies of the transmitter signal $(11.9 \mathrm{kHz})$, the ELF emission, and the upper and the lower sidebands. (b) Three-dimensional plot of the bicoherence spectrum for sum interaction (upper sideband). (c) Same as (b) with contour levels. (d) Three-dimensional plot of bicoherence spectrum for difference interaction (lower sideband). (e) Same as (d) with contour levels.

Each point represents the power value at the relevant frequency, estimated from the Fourier transformation of $\mathbf{5 0 0}$ samples. The powers are expressed in arbitrary units and cannot be compared. It can be seen that the power of the sidebands is increased when the ELF emission is strong and when the transmitter emission is simultaneously decreased. This observation shows qualitatively that the parametric process is operating only when ELF emission is strong enough, and the power in the sidebands can be generated at the expense of the transmitter power. Due to the lack of calibrated data, it was not possible to measure the coupling coefficient for this process, the parameter essential in understanding the energy transfer for the three-wave interaction processes. 


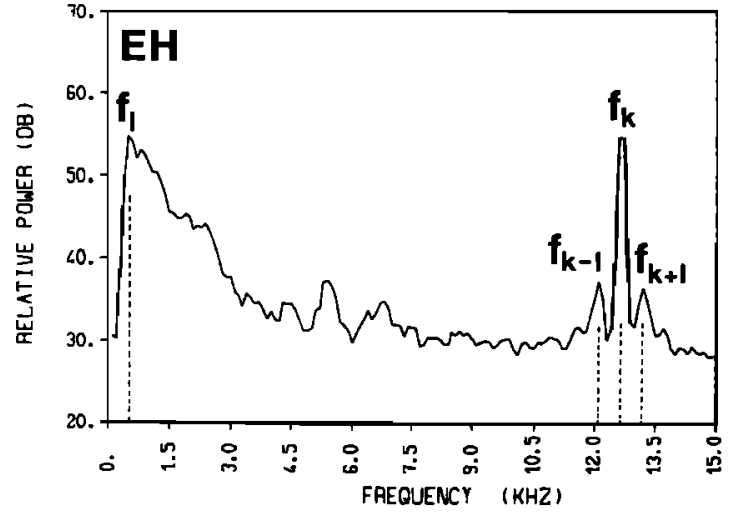

(a)

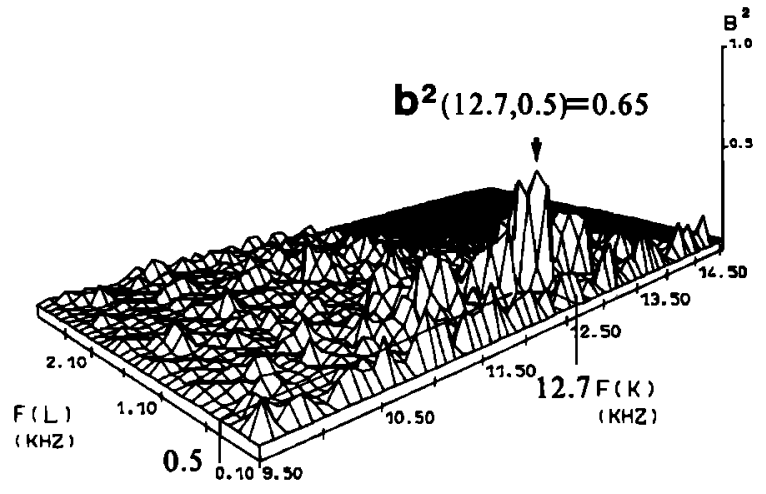

(b)

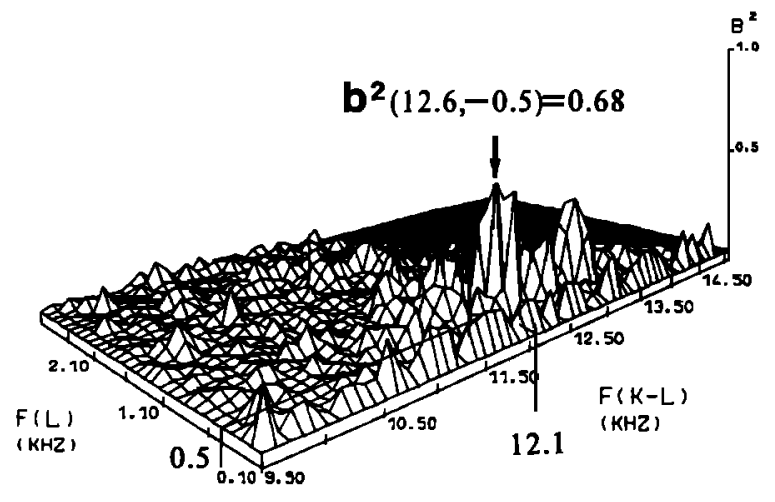

(d)

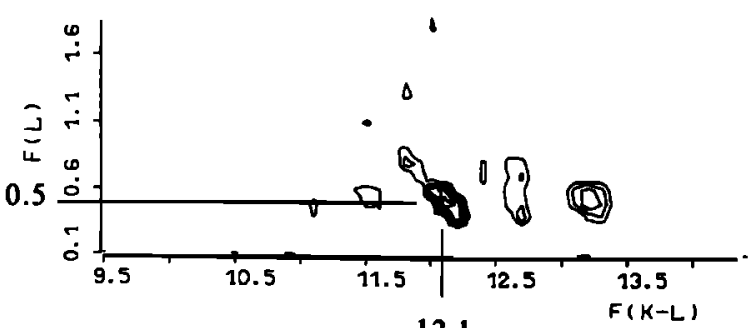

(e)

Fig. 5. Same as Figure 4 at $0423: 28.8 \mathrm{UT}$ and transmitter frequency $12.65 \mathrm{kHz}$. The satellite coordinates are altitude $1191 \mathrm{~km}$, geographic latitude $45^{\circ} 7$, geographic longitude $135^{\circ} 7$.

One has also to be careful with applying the bicoherence analysis in case of slow electrostatic waves received on a moving spacecraft, as their Doppler shift frequencies can no longer satisfy the frequency condition (8). The effect can be insignificant when the bandwidth of spectral analysis or the natural bandwidths of the involved waves are larger than the frequency shift (which is the case here). However, it has been proved by Lagoutte [1983] that even for Dopplershifted frequencies the bicoherence values do not change if the spacecraft is in the source region, i.e., when the wave number condition is still satisfied.

\section{CONCLUSION}

The application of bicoherence analysis to the geophysical data collected in the wave experiment on ARCAD 3 is demonstrated by using two of the five cases of wave interactions at frequencies of the transmitter, ELF emission and the sidebands, as suggested already by Tanaka et al. [1987]. The conclusions from the present study are as follows:

1. The analysis of bicoherence spectra is successful for establishing the phase coherence in the received data at 


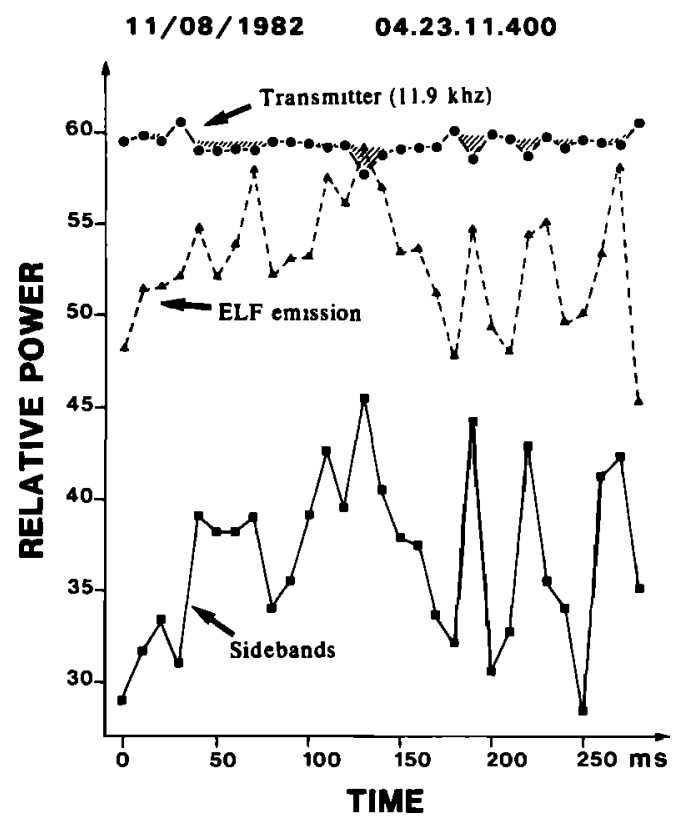

(a)

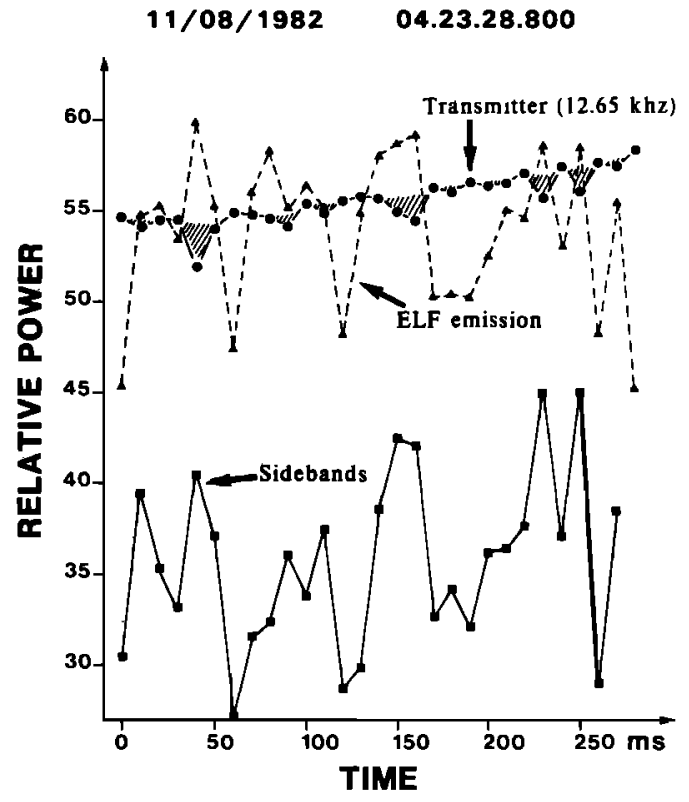

(b)

Fig. 6. Time dependence of powers at the frequencies of the interacting waves. One value of power is obtained by a spectral analysis with $N=500$ samples, which corresponds to 10-ms data. The corresponding symbols are circles for the transmitter, triangles for the ELF emission, and squares for the averaged power of the lower and upper sidebands. The hatched area represents the loss of power of the transmitter. (a) transmitter frequency $11.9 \mathrm{kHz}$, time 0423:11.4 UT. (b) transmitter frequency $12.65 \mathrm{kHz}$, time 0423:28.8 UT.

frequencies of the suggested interaction process. However, to demonstrate its operation in space plasma, information on wave vectors and transfer functions of the receiving equipment is required as well.

2. New and strong confirmation is provided for the parametric interactions of the investigated waves; increased sideband power is observed when ELF emission is high and the transmitter signal is decreased simultaneously.

3. The application of the method faces some limitations, such as the following : (1) The bicoherence estimator depends on the signal-to-noise ratio in such a way that, with decreasing $S / N$, the bicoherence value decreases to zero, even if the coupling in the wave interaction is high. If the signal is stationary over a sufficiently long time interval, one can recover a nonzero value by averaging over a large number of data records. (2) The digitizing of the analog signal has to be done with samples of at least three-bit length, as two-bit sampling produces spurious peaks in the bicoherence spectrum. (3) By definition, the bicoherence analysis is limited to the case of the three-wave interaction (quadratic) only.

4. It is shown that the interpretation of the bispectrum estimator can be misleading, since even for incoherent signals the value of the bispectrum can be significant for large spectral-component amplitudes and a limited number of records $(M)$.

5. While interpreting the bicoherence spectra, one has to be careful about defining interacting waves and a product wave. The confusion of these two in the normalizing denominator leads to an incorrect value of bicoherence.

6. No knowledge is required about a transfer function of a receiver at frequencies of interacting waves, for the estimation of bicoherence; however, this is essential for determinations of the coupling coefficient, bispectrum phase and energy balance in a process of three-wave interaction.

Acknowledgments. We acknowledge J. J. Berthelier as principal investigator and instigator of the ARCAD 3 VLF experiment. We also thank CNES (Toulouse), especially D. Fournier, for the data processing and we are grateful to J. J. Blecki, P. Oberc, and J. Majewski, who first turned our attention to the use of bicoherence in the study of waves in the magnetosphere.

The Editor thanks R. A. Helliwell and another referee for their assistance in evaluating this paper.

\section{REFERENCES}

Altman C., B. Lambège, and A. Roux, Generation of non-thermal E.M. continuum, paper presented at 22nd General Assembly, URSI, Tel Aviv, 1987.

Bendat, J. S., and A. G. Piersol, Random Data: Analysis and Measurement Procedures, John Wiley, New York, 1971.

Berthelier, J. J., F. Lefeuvre, M. M. Mogilevsky, O. A. Molchanov, Yu. I. Galperin, J. F. Karczewski, R. Ney, G. Gogly, C. Guerin, M. Lévèque, J. M. Moreau, and F. X. Sené, Measurements of the VLF electric and magnetic components of waves and DC electric field on board the AUREOL-3 satellite: The TBF- ONCH experiment, Ann. Geophys., 38, 643, 1982.

Elgar, S., and R. T. Guza, Observations of bispectra of shoaling surface gravity waves, J. Fluid Mech., 169, 425, 1985.

Grabbe, C. L., K. Papadopoulos, and P. J. Palmadesso, A coherent nonlinear theory of auroral kilometric radiation, 1, Steady state model, J. Geophys. Res., 85, 3337, 1980.

Huber, P. J., B. Kleiner, T. Gasser, and G. Dumermuth, Statistical methods for investigating phase relations in stationary stochastic processes, IEEE Trans. Audio Electroacoust., 19, 78, 1971.

Kim, Y. C., and E. J. Powers, Digital bispectral analysis and its applications to nonlinear wave interactions, IEEE Trans. Plasma Sci., 7, 120, 1979.

Kim, Y. C., J. M. Beall, and E. J. Powers, Bispectrum and nonlinear wave coupling, Phys. Fluids, 23, 258, 1980.

Koons, H. C., J. L. Roeder, O. H. Bauer, G. Haerendel, R. 
Treumann, R. R. Anderson, D. A. Gurnett, and R. H. Holzworth, Observation of nonlinear wave decay processes in the solar wind by the AMPTE IRM plasma wave experiment, J. Geophys. Res., 92, 5865, 1987.

Lagoutte, D., Analyses spectrale et bispectrale de champs d'ondes électromagnétiques: Modèle AR vectoriel et bicoherence, thèse 3ème cycle, Univ. d'Orléans, Orléans, France, 1983.

Lii, K. S., M. Rosenblatt, and C. Van Atta, Bispectral measurements in turbulence, J. Fluid Mech., 77, 45, 1976.

Riggin, D., and M. C. Kelley, The possible production of lower hybrid parametric instabilities by VLF ground transmitters and by natural emissions, J. Geophys. Res., 87, $2545,1982$.

Roux, A., and R. Pellat, Coherent generation of the auroral kilometric radiation by nonlinear beatings between electrostatic waves, J. Geophys. Res., 84, 5189, 1979.

Tanaka, Y., D. Lagoutte, M. Hayakawa, F. Lefeuvre, and S. Tajima, Spectral broadening of VLF transmitter signals and sideband structure observed on AUREOL 3 satellite at middle latitudes, J. Geophys. Res., 92, 7551, 1987.

Van Atta, C. W. Inertial range bispectra in turbulence, Phys. Fluids, 22, 1440, 1979.

Wu, C.S., and L.C. Lee, A theory of terrestrial kilometric radiation, Astrophys. J., 230, 621 , 1979.

J. Hanasz, Copernicus Astronomical Center, Laboratory of Astrophysics, 87-100 Torun, Chopina 12/18, Poland.

D. Lagoutte and F. Lefeuvre, Laboratoire de Physique et Chimie de l'Environnement, CNRS, 3A, avenue de la Recherche Scientifique, 45071 Orléans Cédex 2, France.

(Received December 29, 1987;

revised April 22, 1988;

accepted April 25, 1988.) 